\title{
Ginecomastia: ¿cuándo estudiarla?
}

\section{Alfredo Jácome Roca}

Internista-Endocrinólogo, miembro honorario de la ACE y miembro de número de la Academia Nacional de Medicina, Bogotá.

Fecha de recepción: 28/03/2017

Fecha de aceptación: 23/06/2017

\section{Resumen}

I ntroducción: La ginecomastia es la proliferación benigna, en el hombre, del tejido glandular mamario que se encuentra debajo del pezón, detrás de la areola. Es necesario diferenciar la pseudoginecomastia de la verdadera, y en ésta, la fisiológica de la patológica.

Métodos: Se hizo una revisión de algunas bases de datos en relación con la ginecomastia patológica, factores endocrinos que influencian el crecimiento mamario masculino, diagnóstico diferencial, proceso diagnóstico y, finalmente, el tratamiento en los casos que así lo requerían. Se buscaron artículos en el periodo 2000-2016.

Resultados: Para resolver la pregunta clínica, en PubMed se encontraron 55 artículos de revisión, y un estudio clínico. En SciELO se encontraron 14 resúmenes de revisiones o series de casos correspondientes a países latinoamericanos. De Colombia, se encontraron 2 artículos. En Lilacs, fueron 6 con las mismas características. Algunos artículos se encontraron repetidos en las bases de datos. En Cochrane, no había referencias sobre el tema.

Discusión: La verdadera ginecomastia es una entidad benigna que pocas veces necesita estudios adicionales. Debe diferenciarse de la pseudoginecomastia, acumulación de grasa subareolar sin proliferación de tejido glandular mamario. Diversas enfermedades se asocian con ginecomastia, particularmente el hipogonadismo, tanto primario como secundario, los síndromes de resistencia androgénica, el hipertiroidismo, la cirrosis hepática y la ingesta de algunos medicamentos. Después de confirmar el diagnóstico, buscando la causa y clasificando la severidad, el manejo debe ser individualizado.

Palabras clave: Ginecomastia, cáncer de seno en hombres, adolescente, obesidad, estrógenos, testosterona, tamoxifeno, aromatasa, cáncer de próstata.

\section{Abstract}

Introduction: Gynecomastia is the benign proliferation of glandular breast tissue in the male, beneath the nipple (subareolar region). Once pseudogynecomastia is ruled out, cases can be classified in physiologic, pharmacologic and pathologic gynecomastia.

Methods: This paper also accounts for a nonsystematic review of some databases in regard to pathologic gynecomastia, factors that influence breast enlargement in the male, differential diagnosis, process of diagnostic investigation and finally, treatment when needed. Searched papers were published between 2000-2016.

Results: To answer clinical question, 55 review articles and a clinical trial were found in Medline, 14 review papers and case reports in SciELO (2 from Colombia), 6 in LILACS, with same characteristics. No reports were found in Cochrane.

Discussion: True gynecomastia is a benign condition than seldom needs additional work-up. A pseudogynecomastia must be ruled out. Several medical conditions are associated with gynecomastia such as primary or secondary gonadal failure, androgen resistance syndromes, hyperthyroidism, chronic liver disease, and the use of some medications. Once diagnosis is confirmed (searching for etiology, staging severity), management should be tailored to each patient.

Keywords: Gynecomastia, male breast cancer, adolescents, obesity, estrogens, testosterone, tamoxifen, aromatase, prostate cancer.

\section{Introducción}

La ginecomastia (GM), definida como la proliferación benigna de tejido glandular mamario masculino asociada con algún depósito local de grasa, es un hallazgo común. Usualmente obedece a un desequilibrio entre la actividad estrogénica tisular y la androgénica ${ }^{(1,2)}$. Una vez se descarta la presencia de una pseudoginecomastia, la verdadera GM se clasifica en fisiológica y patológica, incluyéndose dentro del último grupo unos casos no bien comprendidos que se denominan GM idiopática.

\section{Aspectos básicos del desarrollo mamario}

En la vida fetal temprana, las células epiteliales derivadas de la epidermis del área programada para que posteriormente albergue la aréola, proliferan convirtiéndose en conductos (o ductos) que se conectarán al pezón, que está en la superficie de la piel. Durante la gestación se desarrollarán también, en ambos sexos, unas estructuras alveolares unidas a los ductos. Después del parto, estas estructuras regresan (para volver a 
modificarse en la pubertad), por la caída en los niveles de prolactina, estrógeno y progesterona placentarias ${ }^{(3)}$.

Para que los senos se puedan desarrollar en el individuo prepuberal de ambos sexos, se necesita la presencia de estrógenos (estradiol o E2, estrona o E1, procedentes de ovarios, células de Leydig o de la aromatización periférica de los andrógenos); los estrógenos, ligados al receptor estrogénico (ER), favorecen la proliferación ductal. Se requiere también de progesterona (P) que, ligada al receptor de progestágenos o PR, favorece el desarrollo alveolar. Estas dos hormonas solas no bastan: se requiere también de la indispensable ayuda de las hormonas adenohipofisiarias del crecimiento $(\mathrm{GH})$, la prolactina (PRL), pituitaria y tisular en el tejido mamario, y el factor (tisular) de crecimiento similar a la insulina tipo I (IGF-1) ${ }^{(4-10)}$.

Como decíamos, la unión del estrógeno con su receptor es un factor muy importante en el crecimiento mamario. Este puede deberse a niveles altos de estrógeno circulante de origen endógeno o exógeno, o puede ocurrir por aromatización local de los andrógenos ${ }^{(9,10)}$. Este fenómeno ocurre por acción de la Aromatasa P450, que cataliza la conversión de los esteroides C19 androstenediona, testosterona (TT) y 16-a-hydroxiandrostenediona a estrona (E1), $17 \beta$ estradiol (E2) y estriol (E3), respectivamente. La acción de la aromatasa ocurre principalmente en la grasa intramamaria y subcutánea, pero es vista en otros órganos, aumenta con la edad y con el aumento del índice de masa corporal.

En el sexo masculino, los estrógenos y los andrógenos están equilibrados. Los testículos segregan el 95\% de la testosterona circulante, $15 \%$ del E2 y $5 \%$ de la E1. Para que se presente una GM, se necesita que, o bien haya un aumento del estrógeno circulante o bien una disminución de los andrógenos, que se encargan de bloquear los receptores estrogénicos. Aunque la concentración de estrógenos es muy baja en adultos saludables, estos niveles -en ausencia de andrógenos- pueden causar GM.

La globulina transportadora de hormonas sexuales (SHBG) es una glucoproteína que fija estradiol y testosterona con alta afinidad y especificidad. En general, los esteroides y las hormonas tiroideas se fijan a este tipo de globulinas como la transcortina y la transtiretina, manteniendo una relación con la fracción libre de dichas hormonas, estado que les permite su acción biológica. Su concentración está afectada a la baja por la insulina y el IGF1, y por niveles altos de andrógenos y transcortina; altos niveles de GH, E2 y de T4 incrementan su concentración $^{(10)}$.

La SHBG sirve de marcador en una serie de enfermedades en las que se encuentra alterada su concentración; por ejemplo, las que cursan con hiper e hipoandrogenismo, enfermedades tiroideas e hipofisiarias, hepatopatías y cánceres de próstata y seno. El consumo de alcohol también puede influenciar sus niveles ${ }^{(8)}$. Un metanálisis sobre la relación de TT, testosterona libre (FT), SHBG y síndrome metabólico (MetS), mostró asociación de este último con niveles bajos de SHBG en ambos sexos, de niveles bajos de TT y FT en hombres con MetS y de niveles altos de TT y FT en mujeres con MetS.

Si hay exceso de andrógenos que van a aromatizarse (es decir, a poseer dobles enlaces en los anillos A y B del núcleo esteroide básico), o si hay aumento en la actividad de la aromatasa, se iniciará la cascada del desarrollo mamario en las mujeres, pero también en los hombres. Aunque los andrógenos interceptarían la unión del estrógeno con su receptor, predomina el flujo de estrógenos producto de la aromatización androgénica $^{(5-10)}$.

\section{Revisión del tema}

El objetivo de este artículo consistió en revisar la literatura en los últimos 16 años (siglo XXI) sobre GM de causa patológica o desde la perspectiva del endocrinólogo y definir aquellos casos en que se debe realizar un estudio meticuloso del paciente.

\section{Metodología}

Con el fin de identificar artículos, se realizó una búsqueda en las bases de datos MEDLINE, SciELO, LILACS y Cochrane, utilizando como descriptores de búsqueda los términos "gynecomastia" AND "endocrine" OR "pathologic". Para español, los términos "ginecomastia", "endocrina", "patológica". Se usaron como filtros para depurar la búsqueda, limitándola por años a 2000-2016 para revisiones en idioma inglés o en español, animales humanos de sexo masculino.

\section{Resultados}

Después de descartar resúmenes de artículos duplicados en los términos buscados, en Medline se encontraron 55 artículos de revisión y un estudio clínico. En SciELO se encontraron 14 resúmenes de revisiones o series de casos correspondientes a países latinoamericanos. De Colombia, se encontraron dos artículos. En Lilacs, fueron seis con las mismas características. Algunos artículos se encontraron en las mismas bases de datos. En la base de datos Cochrane, no había referencias sobre el al tema. Una revisión sistemática sobre el tratamiento y la calidad de vida de los enfermos con $\mathrm{GM}^{(11)}$, observó que dichos estudios tenían una muy baja calidad de evidencia, no eran aleatorizados y tenían una alta posibilidad de sesgo. Los artículos considerados más relevantes fueron seleccionados según criterio del autor, para la revisión final.

\section{Pseudoginecomastia}

Al examen del paciente (tabla 1), debe inicialmente descartarse una pseudo GM, aumento de volumen en la zona causado por acumulación de grasa subareolar (lipomastia) sin proliferación glandular; se trata de algo común en personas con sobrepeso, que no requiere valoración posterior ni tratamiento $^{(1,2)}$. 
Tabla 1. Valoración clínica de la ginecomastia*

Historia

- Duración de los síntomas

- Síntomas localizados (masa palpable, dolor o agrandamiento del seno, secreción por el pezón)

- Antecedentes de criptorquidia, paperas, enfermedad hepática o renal

- Recuento detallado de ingesta de medicamentos, suplementos de venta libre, marihuana u otras drogas adictivas, esteroides anabólicos.

- Valoración de la capacidad del estrés ante la presencia del crecimiento de los senos

Examen físico

- Peso y talla

- Signos de feminización según escala de Tanner

- Signos de patología hepática

- Examinar bien los senos y la piel correspondiente

- Presencia de adenopatías regionales

- Examinar la tiroides

- Examinar el escroto y los testículos

${ }^{*}$ Adaptado de Braunstein ${ }^{(1)}$

\section{Ginecomastia fisiológica}

En cuanto a la GM fisiológica, es necesario anotar que, en estos casos como los mencionados en el párrafo anterior, generalmente no es necesario hacer más estudios sino más bien tomar una actitud conservadora de observación. Para tranquilidad -tanto del paciente como del médico- un tema muy importante es la transitoriedad del fenómeno fisiológico, que puede desaparecer desde unos pocos meses hasta en un par de años. Desde la primera consulta, sin embargo, es posible detectar factores etiológicos evidentes como algunos hipogonadismos, enfermedades crónicas y consumos de drogas ilícitas o medicamentos asociados con $\mathrm{GM}^{(12)}$. En los adultos mayores en los que se excluye una acción medicamentosa, el diagnóstico diferencial entre GM fisiológica y patológica puede necesitar una valoración paraclínica, tanto para descartar el poco frecuente cáncer de seno en el hombre como las patologías asociadas, o para concluir que es una GM idiopática y benigna. En los casos que lo ameriten, los exámenes endocrinos usuales son la determinación de E2 sérico (o E1), testosterona total o biodisponible (libre), LH, SHBG, gonadotrofina coriónica humana (hCG), PRL, y pruebas de función tiroidea como la TSH y la T4 libre. Otros exámenes de uso corriente incluyen la ecografía mamaria y testicular, resonancia magnética de hipófisis, pruebas de función hepática y renal, entre otras ${ }^{(13)}$.

Un tamizaje realizado sobre 214 pacientes adultos hospitalizados de entre 27 y 92 años mostró que, aunque había, GM en el $65 \%$, todos eran asintomáticos; la GM se definió como un nódulo de $>2 \mathrm{~cm}$ de diámetro ${ }^{(14)}$.
Como vemos, lo más común es que la GM sea asintomática. Esta es prevalente en 3 picos de edades: recién nacidos (60\%90\% de los casos), adolescentes (50\%-60\% de los casos) y hombres entre 50 y 69 años (hasta el $70 \%$ de los pacientes). La prevalencia de la GM sintomática es mucho menor ${ }^{(2)}$. Los casos restantes son de naturaleza patológica (tabla 2); estos pacientes sí requieren valoración detallada y tratamiento ${ }^{(14,15)}$. Algunas series grandes de casos de GM fueron publicadas en la década de los años $50^{(16)}$.

Tabla 2. Causas de la ginecomastia (*)

\section{Exceso de estrógenos}

A. Estrógenos exógenos: exposición terapéutica o sin intención, incluyendo consumo de andrógenos aromatizables (Vgr. atletas)

B. Estrógenos endógenos. 1. Aumento en secreción testicular (Leydigomas y tumores de células de Sertoli, estimulación de células de Leydig normales por LH o hCG) 2. Aumento en secreción por suprarrenales (tumores feminizantes) 3. Aumento de aromatización de andrógenos a estrógenos (envejecimiento, obesidad, cirrosis alcohólica, hipertiroidismo, medicamentos, tumores secretantes de hCG, síndrome de exceso de aromatasas)

II. Deficiencia de Andrógenos: hipogonadismo primario o secundario, debido a enfermedad, trauma, radiación, o drogas. En el carcinoma de próstata, tratado con antiandrógenos, generalmente se produce GM

III. Relación andrógeno/estrógeno sérica alterada (pubertad, envejecimiento, ginecomastia de realimentación, cirrosis, falla renal y diálisis, hipertiroidismo, drogas)

IV. Disminución de la acción androgénica

A. Antagonistas del receptor androgénico (espironolactona, cimetidina, bicalutamida, flutamida)

B. Receptores androgénicos ausentes o defectuosos.

C. Expansión excesiva de tripletes CAG (citosina-adeninaguanina) en el gen del receptor androgénico (Enfermedad de Kennedy)

*Adaptado de Carlson $\mathrm{HE}^{(16)}$

\section{Ginecomastia neonatal}

En un 70\% de los recién nacidos se puede observar GM(17), resultado de la caída de los estrógenos al finalizar el embarazo, lo que estimula la secreción de prolactina. Esta patología es independiente del sexo del bebé, tiende a ocurrir en la primera semana y desaparece en pocas semanas. Aunque a veces hay secreción, no se recomienda tratar de exprimirlo, ya que puede lograr que permanezca por más tiempo o que se infecte. En algunos casos se puede observar una ginecomastia neonatal gigante (figura 1), indolora, de consistencia firme, no secretante, que igual desaparece en pocas semanas ${ }^{(18)}$. 
Figura 1. Crecimiento gigante de los senos en un recién nacido, que algunos llaman "Mastauxe" (del griego, mastos: senos y auxein: aumento de tamaño)

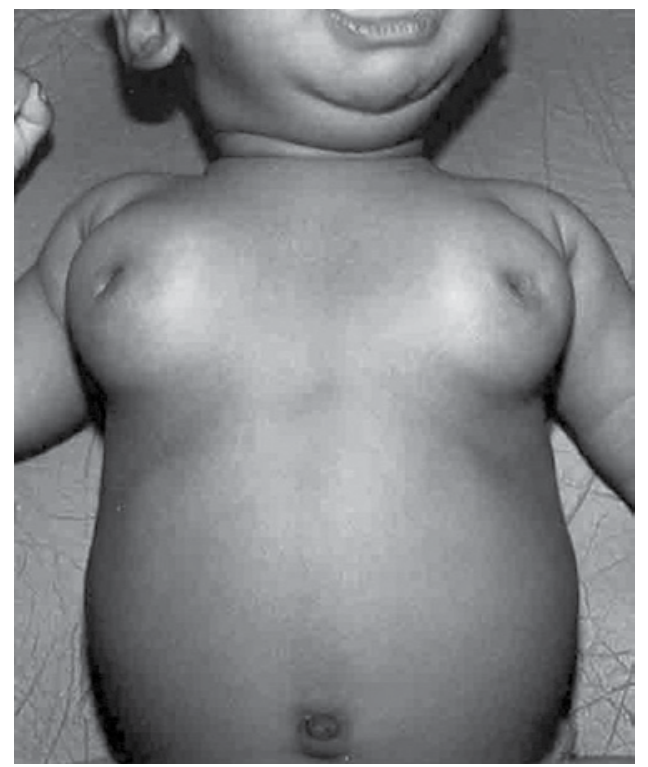

Adaptado de V. Raveenthiran ${ }^{(18)}$

\section{Ginecomastia puberal}

El segundo pico de aparición de la GM ocurre en la pubertad (19-23), pues se puede observar hasta en $60 \%$ de jóvenes a los 14 años, con etapas de Tanner entre II y III (en nuestro medio incluso tiene nombres coloquiales como "piedrilla" o "piedra") (figura 2).

Figura 2. Ginecomastia puberal

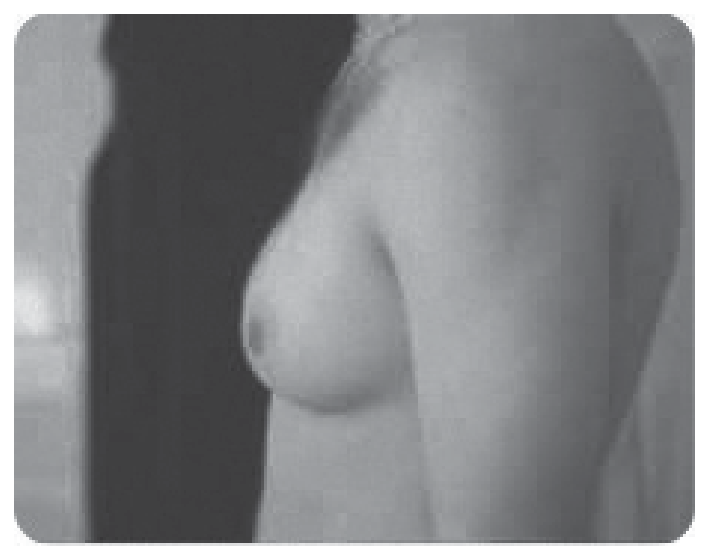

Generalmente se observa como bilateral, aunque a menudo es asimétrica, pero puede ser también unilateral. Usualmente habrá desaparecido dentro de los 3 años siguientes. El mecanismo fisiopatológico involucrado es, ya sea una producción disminuida de andrógenos o un aumento en la aromatización de los andrógenos circulantes, lo que termina generando un aumento en la relación estrógeno/andrógeno. La GM puberal está correlacionada positivamente con la presencia de varicocele, negativamente con el índice de masa corporal y, negativa o positivamente, pero de manera leve, con el volumen testicular ${ }^{(24)}$.

Además de esta causa fisiológica, los adolescentes pueden también presentar GM no fisiológica si consumen alucinógenos, suplementos con anabólicos o ciertos medicamentos. En pocos casos puede ocurrir que una predisposición familiar o genética solo venga a observarse en la pubertad.

\section{Ginecomastia en adultos mayores}

Un porcentaje importante de casos de GM fisiológica puede verse en mayores de 60 años, como resultado de aumento de la aromatasa periférica por exceso de grasa, o por un ligero hipogonadismo presente en los adultos mayores ${ }^{(24,25)}$. La edad también puede generar un aumento de la aromatasa en el tejido graso y se observa además un aumento de la SHBG en este grupo etario. La testosterona se fija mejor a esta globulina transportadora, no así los estrógenos que son menos afines, originando un aumento de los estrógenos libres ante el aumento de la SHBG. Al igual que ocurre en los adolescentes, en los adultos mayores puede observarse una GM patológica, particularmente por la ingesta de múltiples medicamentos formulados.

\section{Nuevos problemas}

No es infrecuente que los atletas que practican deportes de alto rendimiento y los pesistas, se dopen con esteroides anabólicos, gonadotrofinas y $\mathrm{GH}$. Aquellos de sexo masculino no solo desarrollan GM sino que también presentan una supresión de la espermatogénesis ${ }^{(26-28)}$.

La contaminación del ambiente con xenoestrógenos también puede influir; estos disruptores endocrinos son sustancias similares a estrógenos o imitadoras de la función estrogénica, que han venido siendo utilizadas por industrias agroquímicas desde hace varias décadas. Al parecer, causan efectos deletéreos sobre el organismo humano (como la GM), aunque esto es aún motivo de polémica. Entre estos productos están los insecticidas, lociones, preservativos alimenticios, plastificantes, lubricantes, tinturas, emulsificadores, detergentes de laboratorio, pinturas, etc. Dentro de este tipo de productos químicos se destacan los ftalatos, muy utilizados para darle flexibilidad al plástico PVC que es usado, entre otros casos, en juguetes de niños y en los llamados "juguetes sexuales"(29-31).

Carlson $^{(16)}$ suministra un algoritmo que se basa en un cierto número de exámenes para llegar a un diagnóstico de GM idiopática, y no incluye las GM de tipo fisiológico. En esta revisión no incluimos un flujograma, pues para la gran mayoría de los casos no es necesario. En las tablas 2 y $\mathbf{3}$ vemos las causas de la GM. 


\section{Ginecomastia farmacológica}

No sabemos si en todos los casos de medicamentos en los que se ha informado una GM asociada, realmente existe una causa-efecto. No es infrecuente que en la fase de estudio de muchos medicamentos informen GM como hallazgo ocasional, pero generalmente la información queda de ese tamaño. Cuando se presenta esta asociación, generalmente se inicia como una mastodinia unilateral seguida de crecimiento de dicha mama. Solo ocasionalmente, una etiología hormonal puede estar implicada ${ }^{(32-37)}$. En cierto tiempo, la sintomatología y la GM se van reduciendo hasta desaparecer por completo, incluso sin haber suspendido el medicamento, en caso de que sea necesario continuarlo (tabla 3 ).

Tabla 3. Medicamentos asociados con ginecomastia

\begin{tabular}{|c|c|}
\hline Hormonas & $\begin{array}{l}\text { Andrógenos, esteroides } \\
\text { anabólicos, estrógenos, agonistas } \\
\text { estrogénicos, hCG }\end{array}$ \\
\hline $\begin{array}{l}\text { Antiandrógenos/ } \\
\text { inhibidores de la } \\
\text { síntesis androgénica }\end{array}$ & $\begin{array}{l}\text { Bicalutamida, flutamida, } \\
\text { nilutamida, ciproterona, } \\
\text { agonistas de GnRH (leuprolida y } \\
\text { goserelina) }\end{array}$ \\
\hline Antibióticos & $\begin{array}{l}\text { Metronidazol, ketoconazol, } \\
\text { (antimicótico) minociclina, } \\
\text { isoniazida (antituberculoso) }\end{array}$ \\
\hline Antiulcerosos & $\begin{array}{l}\text { Cimetidina, ranitidina y } \\
\text { omeprazol }\end{array}$ \\
\hline Quimioterápicos & $\begin{array}{l}\text { Metotrexate, agentes alquilantes, } \\
\text { alcaloides de la vinca }\end{array}$ \\
\hline Drogas cardiovasculares & $\begin{array}{l}\text { Digoxina, IECAs (captopril, } \\
\text { enalapril), calcioantagonistas } \\
\text { (diltiazem, nifedipino, } \\
\text { verapamilo), amiodarona, } \\
\text { metlldopa, espironolactona, } \\
\text { estatinas, fibratos, reserpina y } \\
\text { minoxidil }\end{array}$ \\
\hline Agentes psicoactivos & $\begin{array}{l}\text { Ansiolíticos (diazepam), } \\
\text { antidepresivos, fenotiazinas, } \\
\text { haloperidol, antipsicóticos } \\
\text { atípicos }\end{array}$ \\
\hline Misceláneos & $\begin{array}{l}\text { Terapia con antirretrovirales } \\
\text { altamente activos, fenitoína, } \\
\text { metoclopramida, penicilamina, } \\
\text { sulindac, teofilina }\end{array}$ \\
\hline \multicolumn{2}{|c|}{$\begin{array}{l}\text { IECA = inhibidores de la enzima convertidora; GnRH = Hormona Liberadora } \\
\text { de Gonadotropina; } \mathrm{hCG} \text { = gonadotropina coriónica. Hay que anotar que varios } \\
\text { de los medicamentos de la lista ya no se utilizan. } \\
\text { Adaptado de Braunstein GD (1) y Goldman RD (33). }\end{array}$} \\
\hline
\end{tabular}

El caso de la GM por espironolactona es diferente. Es usualmente bilateral, indolora, dependiente de la dosis y es mediada hormonalmente. Probablemente representa el comienzo agudo de una verdadera $\mathrm{GM}^{(34,35)}$. Este diurético es un antagonista competitivo de la aldosterona, pero también es inhibidor de la producción testicular de testosterona, aumenta la aromatización a estradiol de dicho andrógeno, y actúa en ciertos tejidos como antiandrogénico, al fijarse a los receptores de andrógenos.

Los mecanismos que inducirían la aparición de GM dependen del medicamento. Las hay con efecto estrogénico (estrógenos, heroína, marihuana, andrógenos, esteroides, anabólicos), gonadotropinas, inhibidores de testosterona (espironolactona, cimetidina, ciproterona, flutamida, clofibrato, omeprazol, ranitidina), mecanismo de retroalimentación (digitálicos, isoniazida), agentes que actúan sobre el sistema nervioso central, que aumentan la PRL (fenotiazinas, antidepresivos), agentes citotóxicos (busulfan, vincristina, nitrosoureas, ciclofosfamida, bleomicina) o finalmente, el mecanismo es desconocido (etionamida, metildopa, reserpina, diazepam, verapamilo, captopril, ketoconazol, metronidazol) ${ }^{(32-37)}$. En $40 \%$ a $70 \%$ de los casos de terapia antiandrogénica para carcinoma prostático aparece una GM como efecto colateral, dependiendo del tipo de droga utilizada y de la duración del tratamiento. Alucinógenos y estimulantes como la cocaína o la heroína se asocian a GM con frecuencia. La marihuana actúa como un fitoestrógeno ${ }^{(37)}$.

Una droga puede ser considerada como causante de GM si hay evidencia significativa obtenida de los estudios publicados. En una revisión que incluyó 92 medicamentos, solo el estudio de 14 de estas drogas (que interferían con la producción o acción de las hormonas sexuales o de la PRL) llevó a un grado A de evidencia. De los estudios de otras 25 se obtuvo evidencia grado B, pero solo en algunas el efecto podía ser esperado por su interferencia en el metabolismo de las hormonas esteroideas o de la PRL ${ }^{(35)}$.

\section{Ginecomastia patológica}

Desde el punto de vista del médico general, este grupo generalmente constituye una minoría. No así para el endocrinólogo, el urólogo, el oncólogo o el cirujano plástico. Este tipo de especialistas a menudo ve en su consulta casos complejos que necesitan investigación adicional o tratamiento médico o quirúrgico. En ocasiones, la GM puede ser de origen multifactorial. La fisiopatología depende de la patología subyacente, si la hay.

\section{Ginecomastia de origen endocrino}

Se ha informado la asociación de ginecomastia con diferentes enfermedades endocrinas, como el síndrome de Klinefelter (hipogonadismo hipergonadotrópico XXY), el hipogonadismo primario, el hipertiroidismo, los defectos en la síntesis de la testosterona, el síndrome de exceso de aromatasa, los tumores de células de Sertoli y variantes del síndrome de insensibilidad androgénica (testículos feminizantes). Estos trastornos tienen hallazgos característicos que permiten una fácil diferenciación de la ginecomastia propia de la pubertad ${ }^{(38-44)}$. 
Lo que ocurre más frecuentemente -principalmente en pacientes obesos- es que los andrógenos se convierten a estrógenos, en virtud de una aromatasa presente en los tejidos, en particular, el adiposo ${ }^{(13)}$. Se observa en pacientes con niveles de testosterona libre disminuidos por hipogonadismo y en síndromes de insensibilidad androgénica ${ }^{(39)}$.

\section{Tumores}

Puede haber elevación de los niveles estrogénicos si se trata de casos como tumores originados en células de Leydig, de células de Sertoli, suprarrenales o los productores de gonadotropina coriónica humana ${ }^{(40,41,43)}$.

\section{Otras causas}

En pacientes con hipertiroidismo, hepatopatías crónicas o ingesta de espironolactona, los niveles séricos de globulina transportadora de hormonas sexuales (SHBG) suelen estar alterados, lo que afecta el balance entre la testosterona libre y la concentración sérica de estrógenos. Sin embargo, todos estos niveles hormonales se encuentran dentro de límites normales en pacientes con ginecomastia puberal, aunque los niveles de testosterona libre son inferiores a los grupos controles ${ }^{(1,9,}$ 12,13,38,44-46). A veces hay trastornos genéticos que afectan los receptores androgénicos, que pueden bloquearse en presencia del quimioterápico bicalutamida, medicamento usado en el tratamiento del cáncer de próstata ${ }^{(47)}$. También puede haber activación del receptor estrogénico con ciertos medicamentos o debido a la presencia de disruptores endocrinos en el medio ambiente.

La enfermedad de Kennedy, también conocida como amioatrofia bulboespinal (BSMA), es una enfermedad rara de la neurona motora, de herencia recesiva ligada al X, caracterizada por una pérdida de masa muscular proximal y bulbar. Solo aparece en hombres. Sus manifestaciones principales son neurológicas, pero entre las no neurológicas están la ginecomastia y el hipogonadismo ${ }^{(45)}$.

\section{Cáncer de seno en el hombre}

El cáncer de seno en el hombre es poco común, solo representa el $1 \%$ de estos tumores y su prevalencia ha sido calculada en 0,7 por cada 100.000 pacientes. No se incluye dentro de los casos de ginecomastia, pero es necesario descartarlo. $\mathrm{Al}$ examen se observa una mama más grande que la otra, presencia de nódulos palpables, generalmente fijos, no móviles, a veces linfadenopatías axilares, puede haber dolor o no, inversión del pezón, secreción líquida, cambios en el color de la piel, etc. En general, la presentación recuerda la de los cánceres de seno en las mujeres ${ }^{(48)}$. Entre los factores de riesgo están la exposición a la radiación, mayores niveles de estrógeno debido a factores como un consumo excesivo de alcohol, cirrosis, obesidad y algunos medicamentos para tratar el cáncer de próstata; la herencia, como antecedentes familiares de cáncer de mama, un gen BRCA1 o BRCA2 mutado y ciertos trastornos genéticos como el síndrome de Klinefelter; ginecomastia previa y la edad, ya que la mayoría de los hombres con cáncer de mama tienen entre 60 y 70 años de edad ${ }^{(9)}$.

Lo correcto para estudiar un caso que lo amerite es hacer estudios imaginológicos y de laboratorio que excluyan endocrinopatías. Cuando resultan normales, se puede hablar de GM idiopática. El laboratorio endocrino incluye la determinación de testosterona total y libre, E2, gonadotropinas hipofisiarias y la coriónica, PRL y pruebas de función tiroidea.

La Clínica Mayo, sede de Jacksonville, Florida, revisó los hallazgos mamográficos de hombres vistos en un quinquenio. Como generalmente se supone, solo el 1\% presentaba cáncer. Las demás causas de masa eran benignas, un $62 \%$ de ellas con un diagnóstico de ginecomastia. Otras patologías fueron lipomas, quistes dermoides y sebáceos, inflamación linfoplasmocítica, ectasia ductal, hematomas y necrosis grasa ${ }^{(49)}$. En una cohorte de 446 hombres que habían sido operados para tratamiento de la GM, hubo una incidencia significativa de cáncer testicular y de carcinoma escamo-celular de piel ${ }^{(50)}$.

Un aspecto muy importante en la valoración de los pacientes es la anamnesis psiquiátrica. Aunque la mayoría de los casos de ginecomastia suelen ser transitorios, un $10 \%$ terminan siendo permanentes, lo que por regla general causa vergüenza en las personas, y en los adolescentes puede ser causa de matoneo en los colegios. Estas personas tienden a no participar en eventos deportivos con el fin de eventualmente ocultar su tórax. Incluso, en los hombres de contextura atlética, la preocupación es evidente, pues son el tipo de individuos que prestan mucha atención a su apariencia física. En los enfermos es posible ver comorbilidades como depresión, ansiedad, bulimia o anorexia, o pérdida de la autoestima ${ }^{(51-53)}$.

\section{Ginecomastia idiopática}

En relación con GM, no menos de 20 enfermedades y 30 medicamentos han sido implicados. Pero la etiología de la GM solo se conoce de manera limitada, por lo que muchos casos -hasta la mitad en algunas series- pueden no tener una causa obvia. Es posible que en estos pacientes juegue algún papel la exposición a un medio ambiente contaminado por disruptores endocrinos con efecto estrogénico.

\section{Clasificación por grados de severidad}

Generalmente se clasifica por grados de I a III, con el paciente en posición de pies ${ }^{(54)}$.

- Grado I: aumento leve, caracterizado por un botón localizado de tejido glandular que se concentra alrededor de la areola, sin exceso de piel en un tórax no adiposo.

- Grado II: aumento moderado. Se caracteriza por una ginecomastia difusa en un tórax con más tejido adiposo, 
con márgenes no bien definidos. Se divide en dos subtipos: a) sin redundancia de piel y b) con redundancia de piel; éste es el grado que generalmente requiere tratamiento quirúrgico.

- Grado III: Gran aumento de la mama. Es una ginecomastia difusa con exceso de piel que requiere resección. Es frecuente y se presenta preferentemente en adultos mayores.

\section{Tratamiento}

El manejo de la GM incluye 3 consideraciones: la observación, la farmacoterapia y la mastectomía. En las personas con ginecomastia reciente habrá que dar un margen de espera, pues el padecimiento puede desaparecer. Una vez analizados todos estos aspectos clínicos y de laboratorio, es necesario tranquilizar al paciente, y suspenderle el medicamento res- ponsable si lo hay y discutir con él alguna patología subyacente. Si se trata de una pseudoginecomastia por acumulación grasa, se puede ofrecer una liposucción.

Usualmente se inicia un tratamiento médico, pero si en un año no ha dado resultados, se acude a la exéresis quirúrgica. La técnica más común es la mastectomía subcutánea, con o sin liposucción. La cirugía es bien tolerada y produce buenos resultados cosméticos. Últimamente se están utilizando técnicas mínimamente invasivas, con mejores resultados ${ }^{(54-56)}$.

En cuanto a medicamentos, los más frecuentemente usados son los modificadores de los receptores estrogénicos como el tamoxifeno y el raloxifeno ${ }^{(57-61)}$. Raramente se han utilizado además la dihidrotestosterona, el danazol, el clomifeno, la testolactona y el anastrazol, estos últimos inhibidores de la aromatasa. La GM asociada con la terapia antiandrogénica para el carcinoma prostático debe prevenirse con tamoxifeno.

\section{Referencias}

1. Braunstein GD. Gynecomastia. N Engl J Med. 2007; 357(12):1229-1237

2. Nuttall FQ. Gynecomastia as a physical finding in normal men. J Clin Endocrinol Metab. 1979; 48(2):338-40.

3. Bocchinfuso WP, Korach KS: Mammary Gland Development and Tumorigenesis in Estrogen Receptor Knockout Mice. Journal of Mammary Gland Biology and Neoplasia 1997; 90: 323-334.

4. Walden PD, Ruan W, Feldman M, Kleinberg DL: Evidence that the Mammary Fat Pad Mediated the Action of Growth Hormone in Mammary Gland Development, Endocrinology 139 (2): 659-62, 1998.

5. Mieritz MG, Sorensen K, Aksglaede L et al. Elevated serum IGF-I, but unaltered sex steroid levels, in healthy boys with pubertal gynaecomastia. Clin Endocrinol (Oxf). 2014;80(5):691-8.

6. LeProvost F, Leroux, C, Martin P Gaye P, Djiane, J, Prolactin Gene Expression in Ovine and Caprine Mammary Gland, Neuroendocrinology 60: 305-313, 1994.

7. Carlson HE, Kane P, Lei ZM, et al: Presence of luteinizing hormone/human chorionic gonadotropin receptors in male breast tissues. J Clin Endocrinol Metab 89(8):4119-23, 2004.

8. Thaler MA, Seifert-Klauss V, Luppa PB. The biomarker sex hormone-binding globulin - from established applications to emerging trends in clinical medicine. Best Pract Res Clin Endocrinol Metab. 2015;29(5):749-60.

9. Swerdloff RS, Ng JCM. Gynecomastia: Etiology, Diagnosis, and Treatment. In De Groot LJ, Chrousos G, Dungan K, Feingold KR, Grossman A, Hershman JM, Koch C, Korbonits M, McLachlan R, New M, Purnell J, Rebar R, Singer F, Vinik A, editors. Endotext [Internet]. South Dartmouth (MA): MDText.com, Inc.; 2000-2015 Aug 3.

10. Barón-Castañeda G, Jácome-Roca A. Aparato reproductor femenino y ciclo menstrual. En Fisiología Endocrina (A. Jácome-Roca, E. Ardila, LA Casas, Eds.), 2017. 4a . Edición, Ed. Manual Moderno, Bogotá.

11. Trost LW, Serefoglu E, Gokce A, Linder BJ, Sartor AO, Hellstrom WJ. Androgen deprivation therapy impact on quality of life and cardiovascular health, monitoring therapeutic replacement. J Sex Med. 2013;10 Suppl 1:84-101.

12. Fagerlund A, Lewin R, Rufolo G, Elander A, Santanelli di Pompeo F, Selvaggi G. Gynecomastia: A systematic review. J Plast Surg Hand Surg. 2015;49(6):311-8

13. Ismail AA, Barth JH. Endocrinology of gynaecomastia. Ann Clin Biochem. 2001; 38(Pt 6):596-607.

14. Niewoehner CB, Nuttal FQ. Gynecomastia in a hospitalized male population. Am J Med. 1984;77:633-638.

15. Nordt CA, DiVasta AD. Gynecomastia in adolescents. Curr Opin Pediatr 2008;20:375-382

16. Carlson HE. Approach to the patient with gynecomastia. J Clin Endocrinol Metab 2011; 96 (1):15-21.
17. Amer A, Fischer H. Neonatal Breast Enlargement. N Engl J Med 2009; 360:1445

18. Raveenthiran V. Neonatal Mastauxe (Breast Enlargement of the Newborn). Neonatal Surg. 2013; 2(3): 31.

19. Johnson RE, Hassan-Murad M. Gynecomastia: Pathophysiology, Evaluation, and Management. Mayo Clin Proc. 2009; 84 (11): 1010-1015.

20. Georgiadis E, Papandreou L, Evangelopoulou C, et al. Incidence of gynaecomastia in 954 young males and its relationship to somatometric parameters. Ann Hum Biol. 1994;21:579-58.

21. Rew L, Young C, Harrison T, Caridi R. A systematic review of literature on psychosocial aspects of gynecomastia in adolescents and young men. J Adolesc. 2015; 43:206-12.

22. Abaci A, Buyukgebiz A. Gynecomastia: review. Pediatr Endocrinol Rev. 2007; 5(1):489-99.

23. Barros AC, Sampaio M de C. Gynecomastia: physiopathology, evaluation and treatment. Sao Paulo Med J. 2012;130(3):187-97.

24. Kumanov P, Deepinder F, Robeva R, Tomova A, Li J, Agarwal A. Relationship of adolescent gynecomastia with varicocele and somatometric parameters: a cross-sectional study in 6200 healthy boys. J Adolesc Health. 2007; 41(2):126-31.

25. Daniels IR, Layer GT. Gynaecomastia. Eur J Surg. 2001 Dec;167(12):885-92.

26. Rahnema CD, Lipshultz LI, Crosnoe LE, Kovac JR, Kim ED. Anabolic steroid-induced hypogonadism: diagnosis and treatment. Fertil Steril. 2014; 101(5):1271-9.

27. Basaria S. Androgen abuse in athletes: detection and consequences. J Clin Endocrinol Metab. 2010;95(4):1533-43.

28. Luis D, Aller R, Cuéllar LA, Terroba C, Romero E. Anabolizantes esteroideos y ginecomastia: Revisión de la literatura. An Med Int. 2001; 18 (9): 45-47.

29. Diamanti-Kandarakis E, Bourguignon JP, Giudice LC, et al. Endocrine-disrupting chemicals: an Endocrine Society scientific statement. Endocr Rev. 2009; 30(4):293-342.

30. Braunstein GD. Environmental gynecomastia. Endocr Pract. 2008;14(4):409-11.

31. Durmaz E, Ozmert EN, Erkekoglu P, et al. Plasma phthalate levels in pubertal gynecomastia. Pediatrics. 2010;125(1):e122-9.

32. Eckman A, Dobs A. Drug-induced gynecomastia. Expert Opin Drug Saf. 2008; 7(6):691-702.

33. Goldman RD. Drug-induced gynecomastia in children and adolescents. Can Fam Physician. 2010 ;56(4):344-5.

34. Nuttall FQ Warrier RS, Gannon MC. Gynecomastia and drugs: a critical evaluation of the literature. Eur J Clin Pharmacol. 2015;71(5):569-7.

35. Krause W. Drug-inducing gynaecomastia--a critical review. Andrologia. 2012 May;44 Suppl 1:621-6. 
36. Deepinder F, Braunstein GD. Drug-induced gynecomastia: an evidencebased review. Expert Opin Drug Saf. 2012; 11(5):779-95.

37. Sauer MA, Rifka SM, Hawks RL, Cutler GB Jr, Loriaux DL. Marijuana: interaction with the estrogen receptor. J Pharmacol Exp Ther. 1983;224(2):404-7.

38. Narula HS, Carlson HE. Gynecomastia. Endocrinol Metab Clin North Am 2007;36(2):497-519.

39. Lanfranco F, Kamischke A, Zitzmann M, Nieschlag E. Klinefelter's syndrome. Lancet. 2004;364(9430):273-83.

40. Forga L, Anda E, Martínez de Esteban JP. Paraneoplastic hormonal syndromes. An Sist Sanit Navar. 2005; 28(2):213-26.

41. Ponce de León Roca J, Algaba Arrea F, Bassas Arnau L, Villavicencio Mavrich H. Leydig cell tumor of the testis. Arch Esp Urol. 2000; 53(6):453-8.

42. Harle LK, Maggio M, Shahani S, Braga-Basaria M, Basaria S. Endocrine complications of androgen-deprivation therapy in men with prostate cancer. Clin Adv Hematol Oncol. 2006;4(9):687-96.

43. Hassan HC, Cullen IM, Casey RG, Rogers E. Gynaecomastia: an endocrine manifestation of testicular cancer. Andrologia. 2008;40(3):152-7.

44. Meikle AW. The interrelationships between thyroid dysfunction and hypogonadism in men and boys. Thyroid. 2004;14 Suppl 1:S17-25.

45. Zajac JD, Fui MN. Kennedy's disease: clinical significance of tandem repeats in the androgen receptor. Adv Exp Med Biol. 2012;769:153-68.

46. García-Benayas T, Blanco F, Martín-Carbonero L, et al. Gynecomastia in HIVinfected patients receiving antiretroviral therapy. AIDS Res Hum Retroviruses. 2003;19(9):739-4

47. Fagerlund A, Cormio L, Palangi L, Lewin R, Santanelli di Pompeo F, Elander A, Selvaggi G. Gynecomastia in Patients with Prostate Cancer: A Systematic Review. PLoS One. 2015;10(8):e0136094.

48. Fentiman IS, Fourquet A, Hortobagyi GN. Male breast cancer. Lancet. 2006; 367(9510):595-604

49. Hines SL, Tan WW, Yasrebi M, DePeri ER, Perez EA. The role of mammography in male patients with breast symptoms. Mayo Clin Proc. 2007; 82(3):297-30.

50. Olsson H, Bladstrom A, Alm P. Male gynecomastia and risk for malignant tumours--a cohort study. BMC Cancer. 2002; 2:26.
51. Ordaz DL, Thompson JK. Gynecomastia and psychological functioning: A review of the literature. Body Image. 2015; 15:141-8.

52. Rew L, Young C, Harrison T, Caridi R. A systematic review of literature on psychosocial aspects of gynecomastia in adolescents and young men. J Adolesc. 2015; 43:206-12.

53. Innocenti A, Melita D, Mori F, Ciancio F, Innocenti M. Management of Gynecomastia in Patients with Different Body Types: Considerations on 312 Consecutive Treated Cases. Ann Plast Surg. 2016 Nov 1. [Epub ahead of print]

54. Marilho Tadeu Dornelas MT, Carvalho-Machado D et al. Surgical treatment of gynecomastia: a critical analysis. Rev Bras Cir Plást. 2010; 25 (3): 470473.

55. Handschin AE, Bietry D, Hüsler R, Banic A, Constantinescu M. Surgical management of gynecomastia-a 10-year analysis. World J Surg. 2008 32(1):38-44

56. Hammond DC. Surgical correction of gynecomastia. Plast Reconstr Surg. 2009;124(1 Suppl):61e-68e

57. Lapid O, van Wingerden JJ, Perlemuter L. Tamoxifen therapy for the management of pubertal gynecomastia: a systematic review. J Pediatr Endocrinol Metab. 2013; 26(9-10):803-7.

58. Devoto C, Enzo; Madariaga A, Marcia; Lioi C, Ximena; Mardones, Nelly. Terapia médica de la ginecomastia con tamoxifeno. Influencia del volumen y duración de la ginecomastia en el resultado terapéutico. Rev Méd Chile, 2007; 135 (12): 1558 - 1565.

59. Costanzo, PR; Suárez, S; Aszpis, SM; Stewart Usher, JG; Pragier, UM; Vásquez Cayoja, M; Iturrieta, S; Knoblovits, P; Pacenza, NA. Ginecomastia: aspectos clínicos y etiológicos. Estudio retrospectivo y multicéntrico Rev Arg Endocrinol Metab, 2015; 52 (1): 22 - 28.

60. Devoto C, Enzo; Madariaga A, Marcia; Aravena, Lucía; Lioi C, Ximena. Etiología de la ginecomastía: Importancia de no subdiagnosticar una ginecomastia patológica. Rev Méd Chile, 2007; 135 (2): 189 - 197.

61. Man Z, Sánchez A, Carretto H, Parma R. Tratamiento de la Ginecomastia con Raloxifeno. Rev Argent Endocrinol Metab. 2003;40:8 -11. 